\title{
Gradhiva
}

GRADHIV

Revue d'anthropologie et d'histoire des arts

$25 \mid 2017$

Gottfried Semper, habiter la couleur

\section{Gottfried Semper et le métabolisme du revêtement architectural}

Gottfried Semper and the metabolism of architectural dressing

\section{Michael Gnehm}

\section{(2) OpenEdition \\ 12 Journals}

\section{Édition électronique}

URL : http://journals.openedition.org/gradhiva/3384

DOI : 10.4000/gradhiva.3384

ISSN : 1760-849X

Éditeur

Musée du quai Branly Jacques Chirac

\section{Édition imprimée}

Date de publication : 31 mai 2017

Pagination : 106-123

ISBN : 978-2-35744-095-1

ISSN : 0764-8928

\section{Référence électronique}

Michael Gnehm, "Gottfried Semper et le métabolisme du revêtement architectural », Gradhiva [En ligne], 25 | 2017, mis en ligne le 31 mai 2019, consulté le 04 janvier 2020. URL : http://

journals.openedition.org/gradhiva/3384; DOI : 10.4000/gradhiva.3384 


\section{Gottfried Semper et le métabolisme du revêtement architectural}

par Michael Gnehm

Les termes de «métabolisme architectural» et de «principe du revêtement » ont une importance majeure dans la pensée de Gottfried Semper. Le regain d'intérêt pour les théories sempériennes - dû, entre autres, à leur élargissement du champ architectural vers les arts industriels dans une perspective globale - refoule toutefois le plus souvent les présupposés de ces deux termes étroitement liés entre eux. Semper les introduit pour rendre compte d'un prétendu progrès historique de l'architecture. Cette approche typiquement historiciste implique, justement de par sa visée globale, une hiérarchisation des cultures prises en considération qui a pour corrélat la justification d'une supériorité des peuples européens. L'analyse proposée ici discute en outre l'interdépendance inévitable entre les théories de Semper et ses projets dans le domaine architectural aussi bien que dans celui des arts industriels. 
Gottfried Semper développe ce qu'il entend par «métabolisme» (Stoffwechsel) en architecture à partir de ses réflexions sur la polychromie architecturale: la peinture, en recouvrant le matériau constructif, fait disparaître ce dernier et le transforme en autre chose que son essence apparente, indiquant ainsi un changement de matériaux. La position de Semper en matière de polychromie et de métabolisme architectural peut être décrite à partir de la recherche française dans le domaine de l'architecture, car Le Jupiter olympien de Quatremère de Quincy (1815) fut pour lui une référence récurrente. Ainsi, le transformisme impliqué dans la notion de métabolisme est étroitement lié, chez Semper, au rapport entre architecture et art industriel - rapport qui pouvait de surcroît être invoqué à travers Quatremère de Quincy, avec par exemple l'illustration du trône de Jupiter. Pour désigner ce processus de transformation impliquant des passages entre artisanat et architecture et, parallèlement, entre différents matériaux, Semper introduisit le terme de Stoffwechsel en 1860 dans le premier volume du Style dans les arts techniques et tectoniques. Stoffwechsel signifie à la fois le "changement de matériaux» et le «métabolisme».

Le changement de matériaux est décrit, depuis Vitruve, en référence au temple grec dans ses relations avec l'origine de l'architecture et la nature; d'abord limité à la relation entre architecture de bois et de pierre, il peut cependant connaître des extrapolations vers l'architecture de fer du XIX ${ }^{e}$ siècle. Semper définit le rapport entre les matériaux en incluant les techniques des arts industriels, du textile, de la céramique et du métal. II formule ce qu'il entend par métabolisme architectural en énonçant son «principe du revêtement »: I'idée que l'architecture est déterminée par son enveloppe extérieure et non par un noyau matériel. L'enveloppe renvoie non pas au matériau constructif réel, mais aux origines architecturales, comme le font un sol en pierre de l'architecture assyrienne ou le plafond d'un temple grec par le biais de leurs ornements dérivés du domaine textile. Comparée à d'autres conceptions du changement de matériaux en architecture, celle de Semper présente une nouveauté: selon lui, l'adoption d'un modèle quelconque, par exemple textile, n'est jamais directe; elle implique toujours la présence d'éléments intermédiaires.

Je voudrais montrer que Semper, en élaborant sa position à partir de la relation entre architecture et arts industriels, se situe dans l'horizon spécifique d'un rapport entre technologie et idéologie, entre une enveloppe architecturale et un certain habitus non seulement social, mais aussi historique. Semper défend une pensée qui évalue les architectures en fonction du degré d'absorption des stades intermédiaires de transformation et de la capacité de l'enveloppe architecturale à rendre justice à l'idée d'un métabolisme architectural, celui-ci étant considéré comme le critère qualitatif d'une comparaison entre les architectures des différents peuples du monde.

\section{La transfiguration architecturale}

Parler de métabolisme architectural emprunt de connotations liées au domaine de la biologie, c'est penser l'architecture comme embrassant toute la vie, y compris la mort. La mort même - illustrée par les monuments funéraires, comme les pyramides égyptiennes, un cercueil assyrien, les tombeaux 
lyciens, le «tombeau de Midas» (cf. supra, p. 89), les urnes ou la dalle funéraire de Bruges - donne une certaine structure aux deux volumes du Style de Semper (1860: 170, 230, 336, 408 et 429; 1863: 13-14, 378, 451 et 529). Les monuments funéraires expriment non seulement le rapport étroit entre arts industriels et architecture, mais encore la façon dont un noyau mort les matériaux constructifs en architecture mis en parallèle avec des cadavres - est animé pour retrouver une vie idéalement éternelle. Cette transposition de la métaphore du métabolisme dans le domaine architectural apparaît dans le passage du Style où Semper définit son objectif comme une tentative pour différencier le principe du revêtement en fonction de plusieurs stades de l'architecture:

La question qui se pose à présent est de savoir ce qu'il est advenu de notre principe du revêtement une fois que s'est accompli le mystère de la transfiguration du projet technico-constructif de l'habitation, en soi tout matériel, en une forme monumentale dont est issue l'art de bâtir ${ }^{1}$ [Baukunst] proprement dit. (Semper 1860: 229²)

Le passage évoqué ici de l'habitation à l'architecture monumentale éclaire la notion de métabolisme architectural: l'un et l'autre impliquent la transformation de quelque chose de tout matériel, l'habitation ou le matériau de départ, en quelque chose d'autre, la forme monumentale ou un matériau secondaire qui renvoie à sa genèse formelle. En ce sens, on peut voir également un parallèle entre les deux métaphores utilisées par Semper, celle du métabolisme architectural et celle du «mystère de la transfiguration ». Toutes deux désignent l'acte de conférer une signification idéelle à l'architecture comprise comme élévation spirituelle aussi bien à l'égard des besoins matériels que des techniques architecturales primaires. Le résultat est que l'apparence matérielle de l'architecture n'est jamais que le signe d'une dimension idéelle. De même que le corps matériel du Christ n'est que l'apparence extérieure d'un mode d'être supérieur en lequel il se transforme, le métabolisme architectural désigne la transformation du matériau potentiellement mort en une spiritualité vivante.

La connotation religieuse n'est pas accidentelle dans la pensée de Semper. Dans le premier volume du Style, il déclare explicitement que «l'art a ainsi le même but que la religion, à savoir de soustraire aux imperfections de l'existence, de faire oublier les souffrances et les luttes de ce monde en laissant entrevoir la perfection » (Semper 1860: XXII, n. 1). La différence qu'il établit entre art et religion permet cependant de préciser la dimension idéologique. Tous deux sont opposés, dit-il, en cela que «la foi, par le mystère du miracle, se plonge dans l'incompréhensible, et par là dans l'informe, tandis que l'art donne forme à l'informe» (ibid.). Or, le «mystère de la transfiguration » actif dans le métabolisme architectural indique que l'art ne se libère pas de toute religion. Mais il s'agit d'une religion spécifique, plutôt protestante que catholique, qui s'apparente à l'idée de Hegel selon laquelle le «principe chrétien » se réalise là «où la liberté prend conscience d'elle-même ${ }^{3}$ ».

Semper vise une transfiguration substitutive et non une transsubstantiation. Son «mystère de la transfiguration » implique l'idée d'une matière
1. C'est Semper qui souligne.

2. Dans cet article, toutes les traductions d'extraits en langue allemande ont été établies par Michael Gnehm et Isabelle Kalinowski.

3. Hegel 1965: 84 (Hegel souligne). Pour la question de la supériorité du protestantisme sur le catholicisme, voir ibid. : 149. 


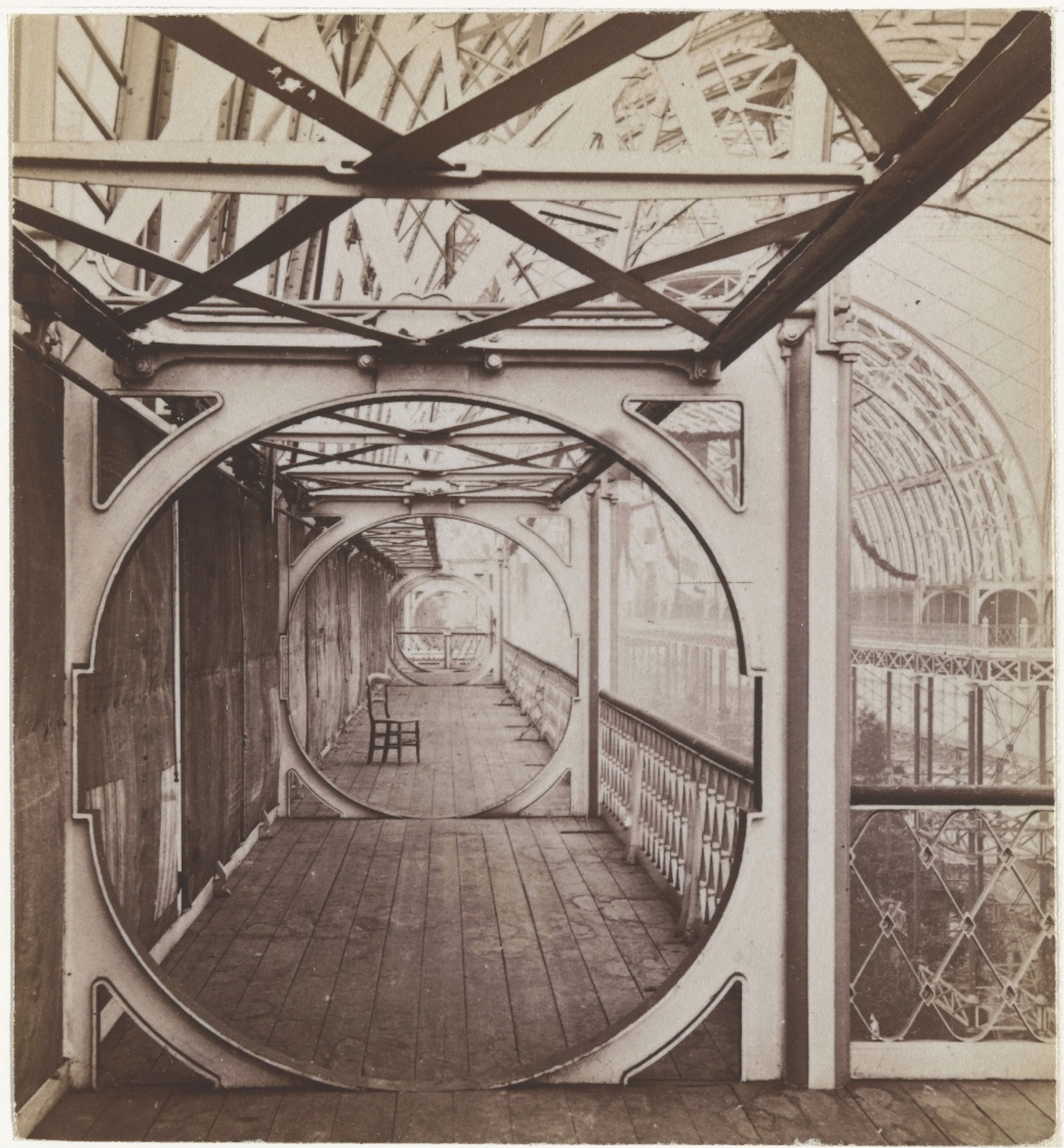


transformée qui préserve l'apparence d'une matière initiale, c'est-à-dire la structure formelle d'un produit de base, comme dans la transfiguration du Christ. En tout cas, Semper semble être opposé à l'idée eucharistique d'une substance indépendante de la matière, dans laquelle cette dernière se transforme en tout autre chose, en ne laissant qu'accidentellement des traces de la matière initiale, comme la substance du pain et du vin devenus corps et sang du Christ grâce à la consécration pendant la messe catholique. Pour Semper, ce qui est substitué persiste ponctuellement après la métamorphose, et se trouve dépassé par ce qui est nouveau, au sens hégélien de l'Aufhebung, aboli et préservé en même temps. Mais il est aussi surmoulé d'une certaine manière, dominé par ce qui est nouveau.

L’idée sempérienne de métabolisme architectural déploie une attitude véritablement historiciste, si on entend par historicisme une idéologie de I'histoire dans laquelle l'histoire se développe comme progrès téléologique, tout en maintenant la nécessité d'enchaîner chaque présent à la totalité de I'histoire. Semper formulait ainsi cette idée dans ses réflexions sur l'Exposition universelle de 1851 à Londres: «Tout était déjà là, sauf que les voies parcourues ne sont pas des courbes finies mais des lignes en spirale, sinon un progrès ne serait pas possible ${ }^{4}$ » (Semper 1851b: fol. 3r; trad. fr in Gnehm 2006-2007 : 69 [trad. modifiée]). Le progrès garantit une issue à ce qui sera chez Nietzsche l'«éternel retour du même», à savoir - écrit Semper dans un de ses derniers textes, prévu pour un troisième volume de Der Stil jamais publié - une issue à «la conformité et [au] retour périodique des événements» (Semper 1870: 257). D'une part, le progrès sempérien comme progrès en spirale assigne aux différents niveaux historiques une place hiérarchique dans le maelström de l'histoire, bien qu'ils puissent faire retour dans chaque présent. D'autre part, la spirale de l'histoire rend possible une réflexivité historique caractérisant le progrès. Ainsi, il existe différents niveaux de développement du métabolisme architectural, qui correspondent à différents niveaux réflexifs d'une prise de conscience de l'architecture par elle-même.

\section{Le textile et le métal}

Le chemin qui conduit à l'architecture passe, selon Semper, par les arts industriels. Leurs matériaux et techniques préfigurent l'expression idéelle de l'architecture. Pour le métabolisme architectural, le textile occupe une place centrale parmi les autres matériaux de la céramique, de la charpente, de l'art du tailleur de pierre et de la métallurgie. Cela tient à des raisons à la fois architecturales et linguistiques. Mis en rapport avec les recherches allemandes sur l'évolution linguistique, le métabolisme sempérien joue sur la parenté langagière entre Gewand, vêtement, et Wand, paroi, qui implique, selon Semper, un processus de changement métabolique du matériau textile vers celui de l'architecture (Semper 1851a: 57; Semper 1860: 229). Bien que les deux termes - issus du même mot winden, tresser - soient apparentés à des verbes différents - Wand à winden mais Gewand à wenden, tourner (Pfeifer [dir.] 1993) -, ils témoignent, pour Semper, d'une certaine immatérialité originelle du mur, Mauer en allemand. Quoi qu'il en soit, la preuve par l'étymologie, aux accents presque heideggeriens, confère au terme de «métabolisme» lui-même, dans sa version allemande, une signification qui est aussi architecturale: le Stoffwechsel, au double sens du mot
4. Une variante de ce passage manuscrit avait été publiée dans Semper 1852: 31 : «Rien de nouveau n'arrive dans le monde; tout était déjà là! Si l'on en croit les philosophes, la société se meut (à supposer qu'elle progresse) selon une ligne en spirale; de certains points de vue, le commencement d'une période coïncide avec sa fin. "

\section{ci-contre}

fig. 1

Henry Negretti et Joseph Warren Zambra, Galerie œil de bœuf, extrême nord de la nef. Album de photographies du Crystal Palace, pl. XVII, 1855-1890. Paris, musée d'Orsay. Photo (c) RMN-Grand Palais (musée d'Orsay) / Hervé Lewandowski. 
5. Bötticher 1852: 33. Voir Wolfgang Herrmann, "Semper und Bötticher", in Herrmann 1981: 26-40; Oechslin 1994; Schwarzer 1995 ; Frampton 2002.

6. Semper souligne.

7. Voir fig. in Semper 1860: 308; et Layard 1840 : pl. XXX.
Stoff, «étoffe» et «matière», associe le processus en question à la transformation du textile en une autre matière.

Selon Semper, dès lors, le textile, motif principal du revêtement architectural, est aussi le médium traversé par chaque métabolisme architectural, y compris celui de l'architecture de bois vers l'architecture de pierre. Semper prend ici position à la fois contre Aloys Hirt et contre Karl Bötticher. L'archéologue Aloys Hirt était l'un des derniers en Allemagne à maintenir l'idée vitruvienne d'une transposition directe des formes architecturales de bois dans le temple grec en pierre. II argumentait d'un point de vue mécaniste en affirmant que la "correspondance entre la construction de bois et celle de pierre» était motivée par la préservation de «principes statiques » (Hirt 1809: 36). L'architecte Karl Bötticher objectait quant à lui que les ressemblances entre les deux constructions étaient dues à la préexistence d'analogies communes auxquelles renvoyaient les «membres» de l'une et de l'autre (Bötticher 1852: 27-28). En se référant aux objets de l'art industriel, Bötticher était proche de Semper. Parmi les analogies préexistantes, il men-

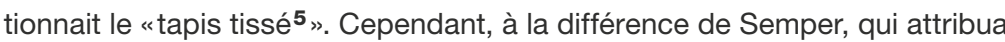
plus tard une importance centrale au tapis, Bötticher rejetait l'idée que ce dernier ait «déterminé l'existence du plafond ou du sol»; il avait seulement «fait naître la symbolique de ces membres » (Bötticher 1852: 33).

Pour Semper, en revanche, «les débuts de la construction coïncident avec les débuts du tissage» (Semper 1860: 227). II n'avance pourtant pas la thèse d'une transposition directe du tissage au bois, ni à la pierre. II affirme certes que les «formes d'art» de la charpente (la tectonique) laissent «entendre un langage artistique connu» «emprunté en majeure partie à l'art textile le plus ancien », et que la «construction en pierre» (la stéréotomie) «entre dans le domaine étendu de l'importante technique » du textile (Semper 1863: 211, 351). Mais Semper voit partout des intermédiaires. Le textile est le point de départ d'autres étapes métaboliques:

Le style de bois a dû être modifié par un changement de matériaux antérieur et ce n'est qu'à partir de cette métamorphose qu'il a pu se transformer en style de pierre, par l'intermédiaire d'un deuxième changement de matériaux ${ }^{6}$. (Semper 1860: 431)

Le missing link, le «chaînon intermédiaire» qui fait le lien entre les constructions de bois et celles de pierre, est le «style métallique» (ibid.: 436).

La preuve en est fournie selon Semper par l'architecture assyrienne. Semper explique le principe du revêtement de cette dernière en évoquant la «tente d'un roi assyrien » tirée du livre The Monuments of Nineveh d'Austen Henry Layard (1849), qui était représentée sur un relief (fig. 2). On pouvait y voir, selon Semper, l'«enrobage des parties constructives de l'édifice ", en l'occurrence « les parois en bois, les poteaux et plafonds revêtus de métal ${ }^{7}$ " (ibid.: 302). Cet enrobage métallique renvoyait au «principe de la construction tubulaire», autrement dit à la technique consistant à envelopper de métal une partie constructive comme le poteau de bois, avec pour effet de rendre le noyau superflu, du fait de la stabilité matérielle de cette enveloppe. 


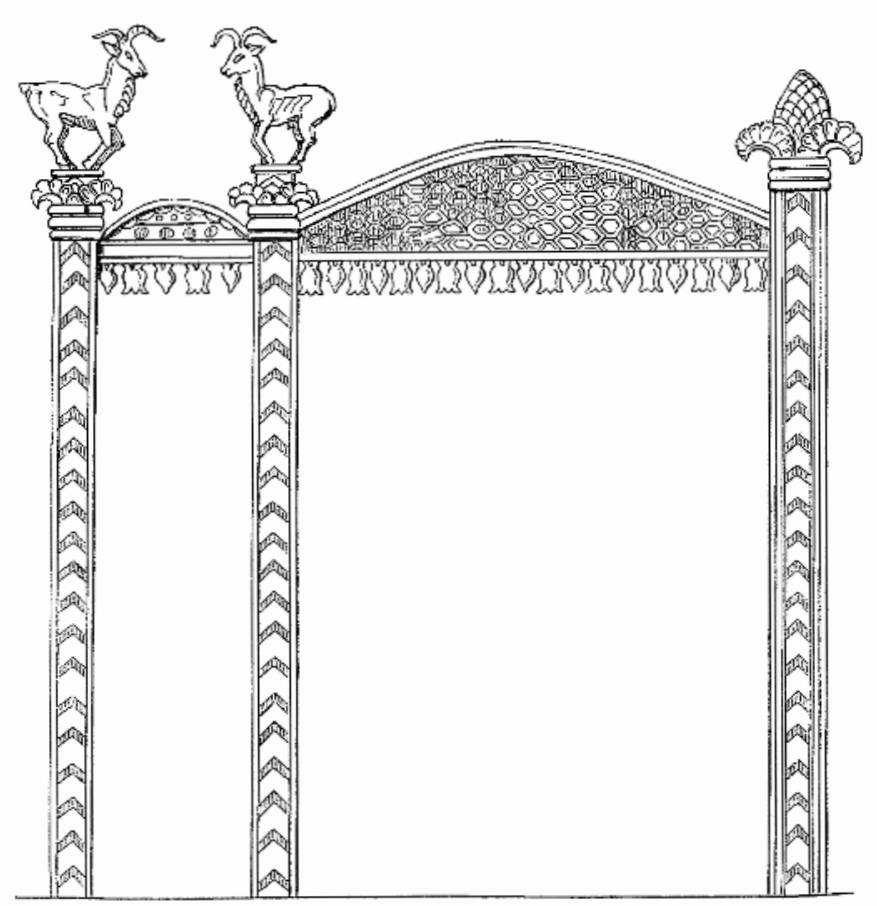

La construction tubulaire en métal des Assyriens devient, pour Semper, la référence de son renversement, de sa «transvaluation» de la tectonique traditionnelle. Selon lui, l'apparence du temple grec a été tributaire de cet intermédiaire métallique entre bois et pierre. Semper en voit la preuve dans un «ornement architectural mycénien» du Trésor d'Atrée (fig. 3). Les «ornements en zigzag et en spirale» de «fûts en marbre avec leur plafond décoratif général » renvoient bien au textile. Mais, comme il put s'en convaincre en examinant les originaux conservés au British Museum, ces «fûts exécutés en marbre " correspondaient également à des "fûts bosselés en métal ${ }^{8}$ " (ibid. : 439).

\section{Material agency et spiritualisation}

Le métal est le matériau qui, selon Semper, a pris la relève du textile. Ainsi, il dit du métal qu'il «englobe toutes les branches de la technique grâce à la malléabilité de sa matière " (Semper 1863: 479). Le métal est quasiment du textile solidifié. Or, si le métal ne se contente pas de recouvrir les autres techniques mais intervient également dans les changements de matériau entre bois, textile et pierre, il paraît agir toujours dans le sens d'un déterminisme matériel, tout en atténuant l'influence d'autres matériaux. Cependant, l'exemple des fûts mycéniens - dressés en marbre tout en renvoyant au métal - démontre pour Semper l'importance de la capacité à surmonter une matière initiale, déterminante pour toute la technique architecturale. L'exploit des Grecs est d'avoir intégré toutes les étapes différentes du changement de matériaux dans leurs constructions. À la transposition de formes textiles dans des formes de bois et de ces dernières dans des formes de pierre, les Grecs ont ajouté un troisième niveau de transformation, celui de la réflexion sur les processus de changement de matériaux. À partir de la «construction de corps creux», de la construction tubulaire, les Grecs ont «interprété cette idée constructive non pas de manière réaliste, mais dans un sens supérieur ", tout en ayant pris «pleinement conscience de soi » (Semper 1860: 437-438). fig $=2$

Gottfried Semper, Tente d'un rois assyrien, in Der Stil, t. I, 1860.

fig. 3

Gottfried Semper, Ornement du Trésor d'Atrée, in Der Stil, t. I, 1860.

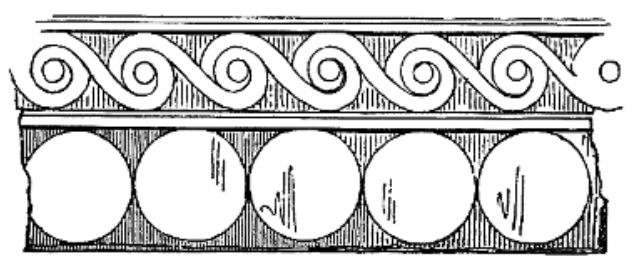

8. Semper avait examiné "les lambris de marbre richement décorés et le morceau d'une colonne provenant du soi-disant Trésor d'Atrée à Mycènes " au British Museum en 1852 au plus tard, comme il l'explique dans un article paru en 1855 (Semper 1855 : 406). Voir le projet d'une «Décoration festive pour le $80^{\mathrm{e}}$ anniversaire du roi Anton " de 1835 (Nerdinger et Oechslin [dir.] 2003: 157-158), qui comporte une colonne à ornement en zigzag. Peut-être Semper connaissait-il déjà alors The Antiquities of Athens de 1830 de Charles Robert Cockerell, publication qui contient des illustrations représentant le Trésor d'Atrée. 
9. Voir «Gotik", in Herrmann 1981: 23-24
Cette prise de conscience les distingue des autres peuples, comme Semper croit pouvoir le constater en étudiant notamment l'exemple de l'architecture en Inde, c'est-à-dire dans l'Empire des Indes britanniques.

Pour Semper, l'architecture indienne fournit l'illustration que le stuc est, "depuis des temps immémoriaux, un des agents les plus importants de l'architecture et de la sculpture orientales». Le stuc est le «médium matériel qui a favorisé [leur] richesse figurative surchargée». Le surmoulage en stuc fait partie des «différents changements de matériaux » qui ont précédé, comme dans le cas des pagodes, la «transformation du style de bois en un style monolithique» (fig. 4) [ibid. : 259]. Le stuc est, en fait, l'antimatière du métal: aussi malléable que ce dernier, il se prête surtout à la création d'un moulage, c'est-à-dire d'un récipient vide - vide de sens dans la perspective de Semper. Car, pour lui, l'architecture indienne représente une des antithèses de l'architecture grecque. L'architecture monumentale en pierre des Indes et de l'Orient en général n'a vu le jour qu'au moment où «l'art était achevé depuis longtemps ». Par contraste, l'architecture monumentale des Grecs n'a trouvé sa forme artistique «qu'avec le temple de pierre» (ibid.). Elle démontre, selon Semper, qu'on ne doit pas laisser libre cours à la matière comme agent. Avec la pierre, la matière se présente encore sous une forme récalcitrante dans l'architecture grecque, mais celle-ci se montre capable, grâce à son "sens supérieur » de l'autoréflexion sur sa genèse, de surmonter le caractère lapidaire de son matériau d'art. L'architecture grecque, contrairement à celle de l'Orient, n'est pas victime de la material agency, pour emprunter un terme à la mode qui renvoie, entre autres, aux travaux d'Alfred Gell et de Bruno Latour et qui désigne - dans sa conception simpliste - l'idée que des «objets agissent de leur propre volonté » (Jones et Boivin 2010: 334).

En se laissant submerger par la matière, l'architecture orientale a suivi le même chemin que l'art industriel d'Asie tel que Semper le décrit dans sa brochure Science, industrie et art, à l'occasion de la première Exposition universelle de 1851 à Londres. Semper y fait état d'une admiration sincère pour «l'industrie d'art extra-européenne», surtout celle des Indiens orientaux. Dans le même temps, cependant, il décèle dans les «exploits des Asiatiques" l'absence d'une "expression individuelle, d'un langage, d'un beau phonétiquement supérieur, d'une âme » (Semper 1852: 11 et 25). Les arts industriels des Orientaux ne fournissent pour Semper qu'une grammaire, mais non un langage vivant, un ton individuel. En échouant à doter la matière d'une âme, ils échouent à transformer ce qui était potentiellement mort en un être vivant. Bref, ils échouent à concevoir le changement de matériaux comme un métabolisme dont le but est la spiritualisation autoréflexive.

Dans le modèle historiciste de Semper, toutefois, il n'est qu'une architecture pire que celle de l'Orient: c'est l'architecture gothique, au moins à partir de l'échec qu'essuya en 1845 son projet présenté au concours pour l'église Saint-Nicolas de Hambourg, construite finalement par George Gilbert Scott comme une église néogothique. Pour Semper, le style gothique n'était plus que l'expression de la propagande catholique de son temps ${ }^{9}$. Il le punit en ne donnant aucune illustration de cathédrale gothique dans Le Style, comme pour faire contrepoids aux publications d'Eugène Emmanuel Viollet-le-Duc. 


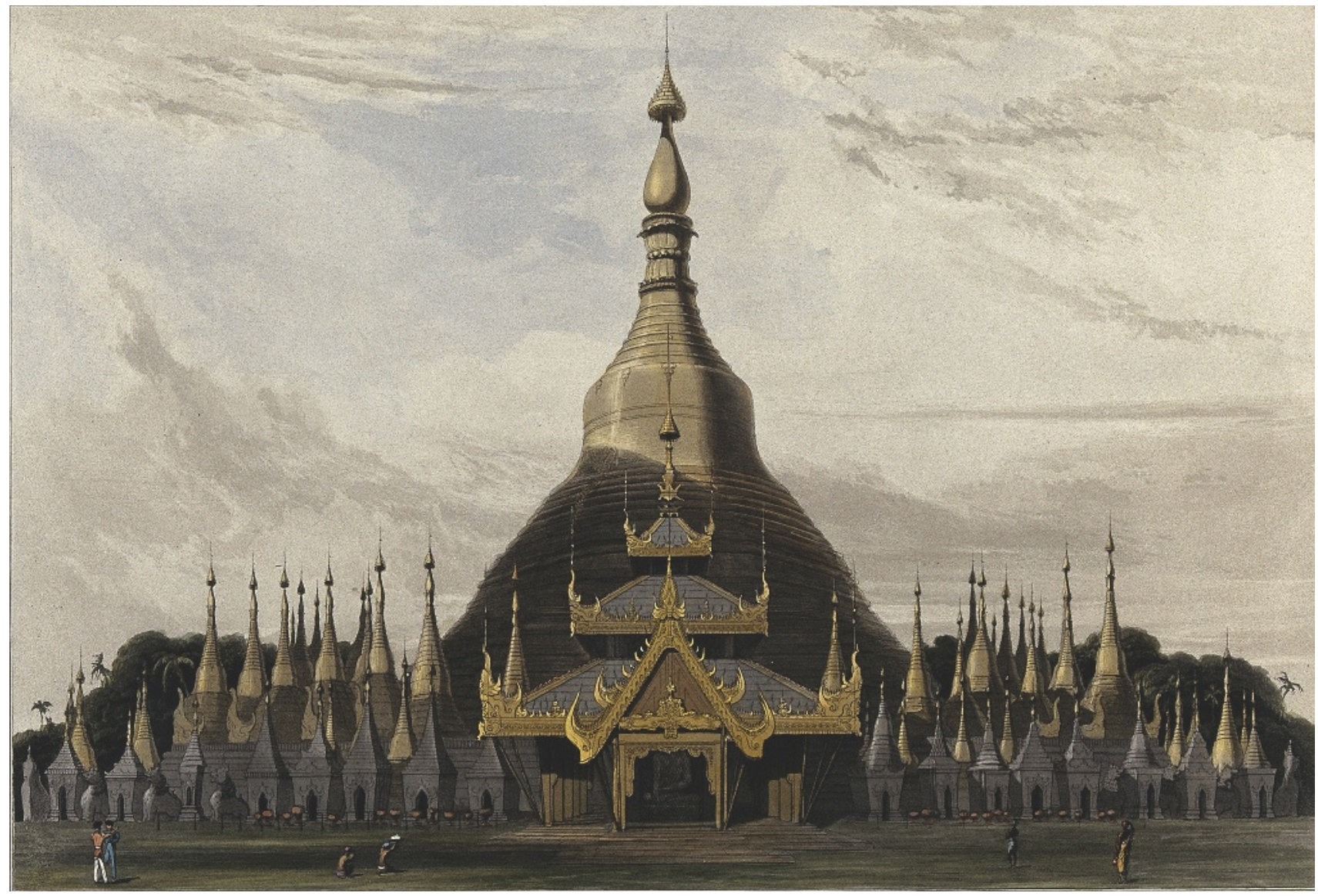

L'architecture gothique, dit Semper en commentant le Dictionnaire raisonné de l'architecture française de Viollet-le-Duc, «ne veut rien savoir du revêtement, parce que son élément est justement l'apparition nue des parties fonctionnelles, car elle doit faire parade, comme l'écrevisse cuirassée, de son squelette tout en le montrant en pleine activité " (Semper 1860: 320, trad. fr. in Gnehm 2006-2007: 67 [traduction modifiée]). Un squelette en activité sous-entendant donc un spectre, ou un animal, mais non un être humain, et, traduit en architecture, la pierre ou le métal morts. C'est dans ce sens que Semper voit dans les cathédrales gothiques une réalisation du "principe technique et matérialiste». Celui-ci, selon les mots de Semper, était adopté «symboliquement dans le sens d'un châtiment de la matière". Les cathédrales gothiques tentaient de s'abstenir de la matière tout en la torturant. Pour Semper, l'architecture gothique est opposée à la spiritualité chrétienne qui contenait, "selon la doctrine de la crucifixion de la chair du Christ, [...] en même temps sa reconnaissance" (ibid.: 506). Spiritualisation et, dans le même temps, reconnaissance de la chair: tel semble être le paradoxe sempérien.

\section{Exemples pratiques}

J'ai voulu esquisser ici la position de Semper comme à la fois anticatholique et colonialiste, c'est-à-dire classant les sociétés en fonction de leur degré de réalisation d'une idée transcendante. Reste l'épreuve de la pratique. Je donnerai quelques illustrations des formes de synthèse que Semper a imaginées pour le domaine de l'architecture contemporaine en rapport avec les arts industriels. En 1858, Semper a dessiné d'une part fig. 4

William Daniell, d'après James Kershaw, Pagode de Dagon, près de

Rangoun, Myanmar (Birmanie), aquatinte, vers 1831, éd. Smith, Elder and Co., Londres: [1831], 35 x 47,8 cm. 


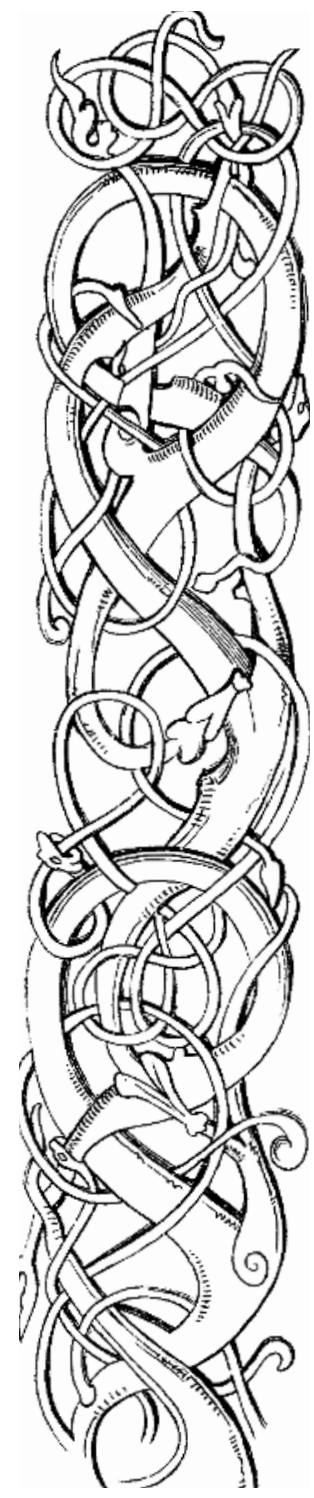

fig. 5

Gottfried Semper

"Scandinave", in Der Stil,

t. I. 1860 , p. 83.

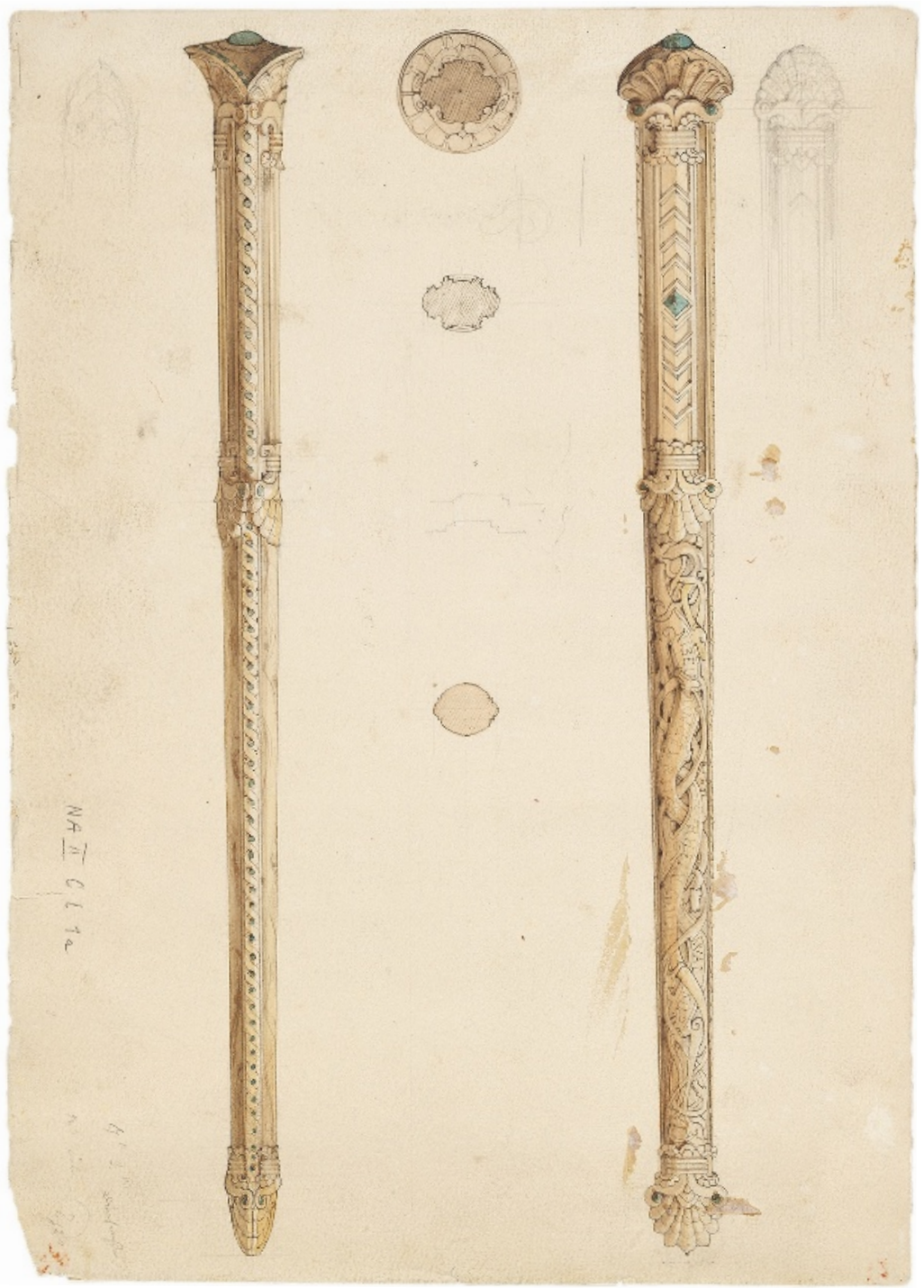

fig. 6

Gottfried Semper, Bâton

de chef d'orchestre pour

Richard Wagner, 1858.

Nationalarchiv der Richard-

Wagner-Stiftung, Bayreuth. 
une baguette de chef d'orchestre pour Richard Wagner (disparue depuis la fin de la Seconde Guerre mondiale). L'idée de ce cadeau imaginé par Mathilde Wesendonck était de représenter un «bâton runique» (fig. 6). Avec une longueur estimée de $37 \mathrm{~cm}$ et un poids par conséquent assez considérable, ce «bâton » remplissait une fonction moins pratique que symbolique; il représentait moins une baguette qu'un sceptre et par suite un objet cérémoniel (Kötzsche 1983; Schröder 1990). D'autre part, Semper a participé la même année à un concours pour la construction d'un Opéra impérial à Rio de Janeiro - projet qui préfigurait celui d'un Opéra pour Richard Wagner à Bayreuth (fig. 7) [Laudel 2003]. Les deux projets portaient la marque d'une hybridité que Semper poursuivait à cette époque - hybridité entendue comme une sorte de synthèse qui devait abolir et préserver (aufheben) un paradoxe possible dans sa pensée ${ }^{\mathbf{1 0}}$.

La baguette imaginée pour Wagner mêlait deux ornements adoptés de différentes cultures. La partie supérieure reprenait l'ornement figurant sur les poteaux de la tente assyrienne interprétée par Semper comme revêtue de métal (fig. 2). La partie inférieure de la baguette était formée d'après un ornement de bois du xII ${ }^{\mathrm{e}}$ siècle issu d'un portail de la stavkirke d'Urnes en Norvège (fig. 5) [Semper 1860: 83; 1863: 285]. Cet ornement nodulaire représentait selon Semper «le chaos même » et le «symbole de l'enchaînement primitif des choses » sous un «rapport mystico-religieux». Remplissant cette fonction, ce nœud formait la base de toutes les formes ornementales dans un sens «constructif et actif» (Semper 1860: 83-84).

Or, la baguette wagnérienne fut exécutée en ivoire. On peut établir un parallèle entre cette matérialité et le changement de matériaux chez les Grecs, qui transformaient en pierre les modèles de bois, par l'intermédiaire du métal. Le nouveau matériau, en l'occurrence l'ivoire, greffait deux traditions sur la baguette, celles des Assyriens et des Vikings. Les deux éléments étaient présentés dans leur interdépendance, comme l'indiquaient les palmettes assyriennes qui articulaient et délimitaient la baguette. Or, l'art assyrien était perçu comme une des bases de l'art grec, et, de la même façon, l'art scandinave comme un des fondements de l'art germanique. On retrouve ici ce que Semper formulait comme le projet de son Style dans les arts techniques et tectoniques: démontrer la «relation primitive entre les deux peuples indo-germaniques, les Grecs et les Germains » (Semper 1863: 290 et 265).

Enfin, la baguette de chef d'orchestre a pour fonction de contrôler ce qui se produit sous sa direction, c'est-à-dire une musique qui possédait tout ce qui manquait à l'art oriental: «l'expression individuelle, un langage, un beau phonétiquement supérieur, une âme» (Semper 1852: 25). Son corps de résonance est le bâtiment lui-même. L'Opéra que Semper dessine pour Rio de Janeiro se distingue par une certaine hybridité qu'il partage avec la baguette de Wagner. Semper prolonge les pilastres extérieurs de la salle en piliers dégagés surmontant l'entablement et associés à des arcs-boutants sur lesquels prend appui la structure en fer du toit de la salle (fig. 8) [Bayer 1894: 18]. Ce mélange d'éléments architecturaux issus de la Renaissance et du Moyen Âge est extraordinaire au sens où l'aspect manifestement le plus technique - la structure en fer du toit de la salle - est rendu visible
10. Semper parle d'«hybride archaïque » pour désigner le «mélange de parties doriques et ioniques et d'éléments barbares [...] de la période grecque moyenne " et la "colonne du temple d'Héra à Samos " (Semper 1860: 438). 

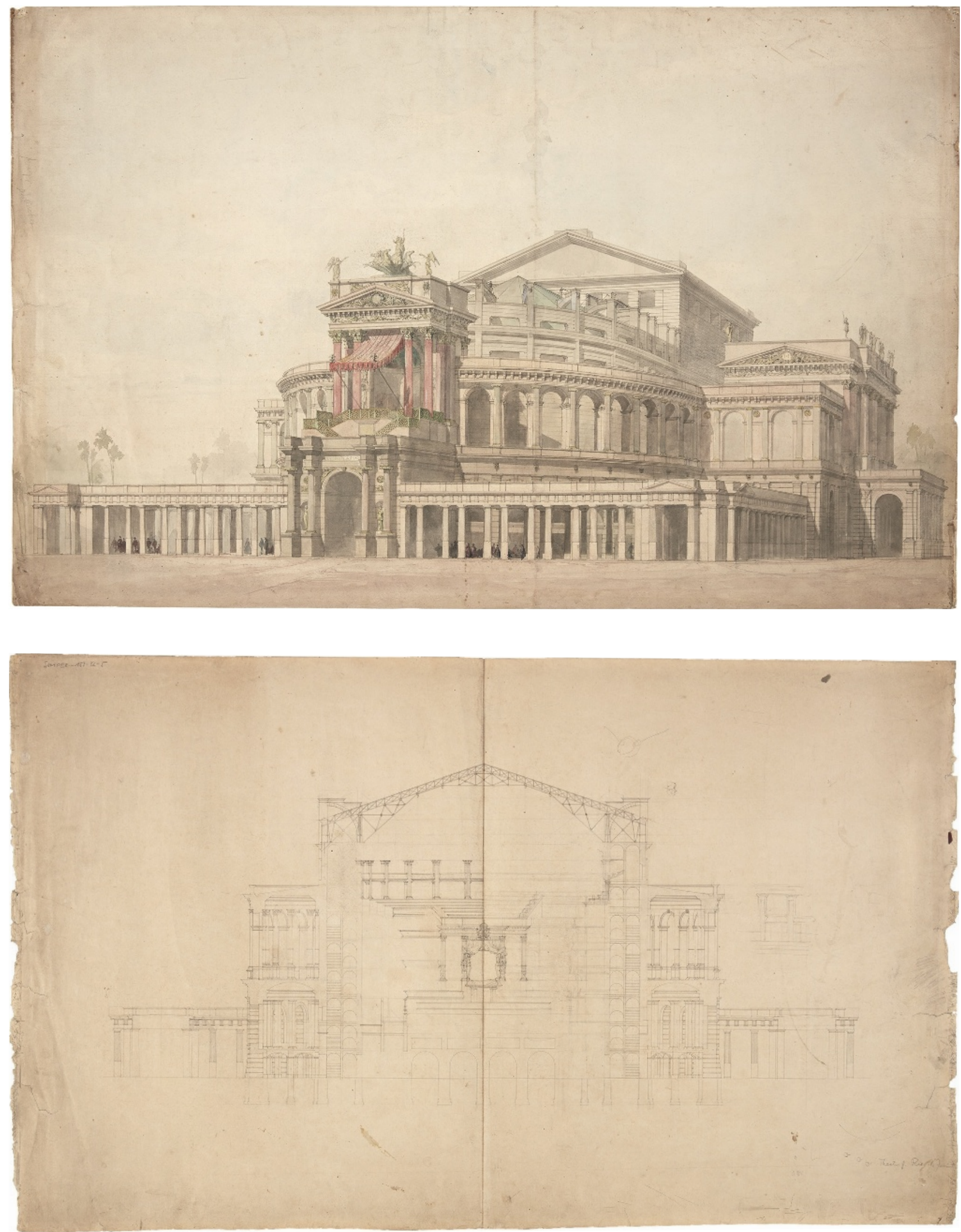


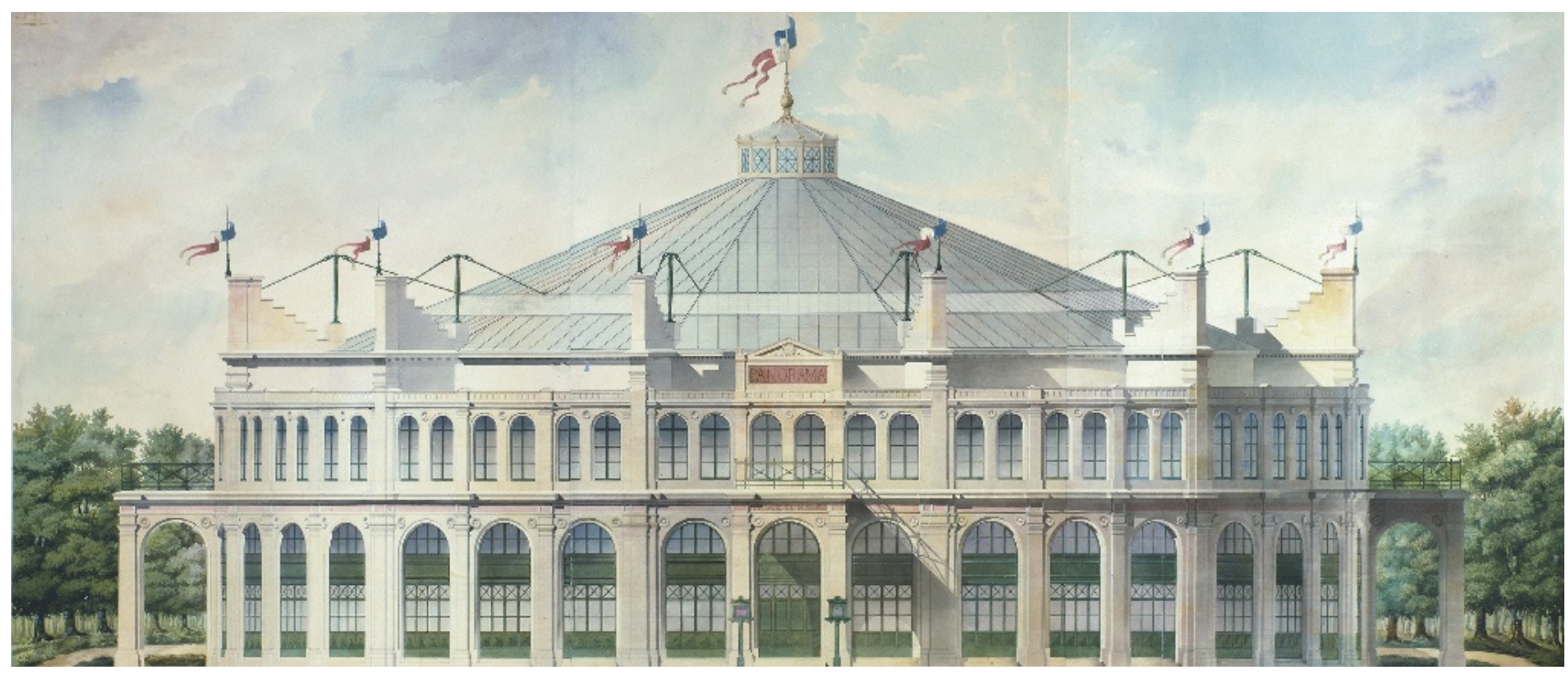

fig. 9

Jakob Ignaz Hittorff,

Champs-Élysées,

Grand Carré, la rotonde

des Panoramas

1838-1839, aquarelle,

crayon, 66,2 x 138,2 cm.

Cologne, Wallraf-Richartz-

Museum \& Fondation

Corboud, Inv.-Nr. Ch. 686a.

Bildnachweis: Rheinisches

Bildarchiv Köln.

\section{ci-contre}

fig. 7

Gottfried Semper, Projet

d'opéra pour Rio de Janeiro,

1858, perspective. Deutsches

Theatermuseum Munich,

Inv. Nr. : VII 3475 (F6043)

Photo (C) Rudolf Faist.

fig. 8

Gottfried Semper, Projet

d'opéra pour Rio de Janeiro,

1858, coupe. Deutsches

Theatermuseum Munich,

Inv. Nr. : Semper 153-32-5

(F9061). Photo () Rudolf Faist. 


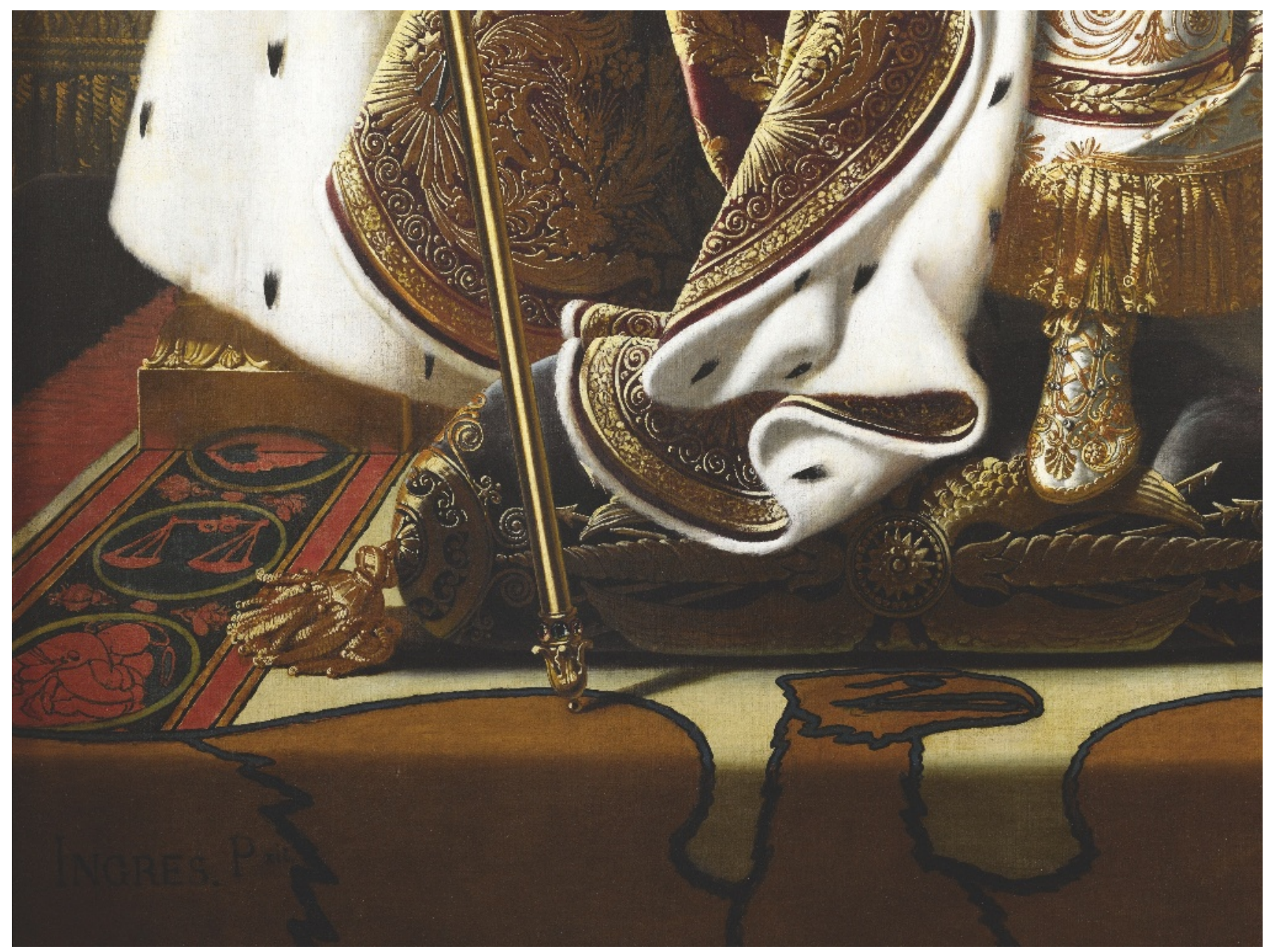

fig. 10

Jean-Auguste-Dominique

Ingres, Napoléon ler sur

le trône impérial ou

Sa majesté l'Empereur

des Français sur son trône,

1806, détail. Huile sur

toile, $260 \times 163 \mathrm{~cm}$, Paris,

musée de l'Armée.

Photo (C) Paris - Musée de

l'Armée. Dist. RMN-Grand

Palais / Emilie Cambier. 
à l'extérieur par les tirants métalliques en combinaison avec les arcs-boutants - éléments emblématiques de l'architecture gothique qui désigne, pour Semper, un matérialisme primordial et primitif en architecture.

Cet élément technique rendu visible est manifestement un tribut de Semper au pays auquel l'Opéra était destiné: le Brésil, comme lieu exotique et primitif dans ses fondements. Mais la conception de l'hybridité de Semper ne s'arrête pas là. La rotonde des Panoramas de Jacques Ignace Hittorff à Paris, qui servit visiblement de modèle pour l'Opéra brésilien de Semper, permet d'élucider ce point (fig. 9). Les arcs-boutants de Hittorff, conçus en gradins massifs, possèdent une forme plus médiévale que ceux de Semper; ceux-ci sont plus réduits et plus plats, et percés par l'insertion d'un arc en quart de cercle. De plus, la façade de la salle de l'Opéra est elle-même disposée en gradins: certaines parties sont situées devant celle dont les pilastres se terminent en arcs-boutants. Les parties antérieures, les portiques rectangulaires du rez-de-chaussée, forment, selon Semper, un «rideau transparent de [...] colonnade aréostyle» qui fait apparaître l'édifice comme «plus grandiose» encore par le contraste d'échelle (Semper 1858: fol. 3v). Ce caractère appelé «grandiose» est justement dû à la montagne en terrasses de colonnades et d'arcades successives qui finissent par former un rideau opaque cachant la structure intérieure en fer et ne laissant transparaître celle-ci que secondairement, sous la forme d'arcs-boutants qui disparaissent encore devant la tour scénique. La théâtralité de cette construction manifeste sous une forme performative et dynamique le «principe du revêtement » en soumettant tout ce qui est conçu comme «primitif » à une spiritualisation que Semper appelle métabolique.

Le regain d'intérêt pour Semper dans l'histoire de l'art et la théorie de l'architecture depuis à peu près une dizaine d'années tient avant tout aux implications supposées des termes sempériens discutés ici. Semper est souvent perçu comme un précurseur héroïque en matière théorique et méthodologique car il a semblé anticiper la réflexion sur des sujets qui ont marqué d'une part le modernisme avec, par exemple, son goût pour le «primitivisme», et d'autre part le postcolonialisme avec sa réévaluation de I'histoire de l'art sous un angle global. En revanche, j'ai essayé de montrer comment les termes du métabolisme architectural et du principe du revêtement évoquent un contexte aussi bien théorique que politique profondément ancré dans le $x \mathrm{X}^{\mathrm{e}}$ siècle. Sans vouloir dévaluer l'originalité de la pensée de Semper, il me semble impératif de tenir compte d'un horizon théorique abordé dans une perspective historiciste qui incarne, au sens hégélien, l'idée transcendante du beau dans une matérialité transfigurée. En cela, cette perspective s'inscrit dans le cadre colonialiste de l'époque de Semper: ses jugements sur l'ensemble des arts des différentes sociétés occidentales et orientales - révèlent qu'il concevait celles-ci selon un ordre hiérarchique qui positionne les acquis occidentaux à la tête d'un prétendu progrès historique. Ainsi, la théorie sempérienne pourrait bien se présenter comme l'inconscient refoulé de notre temps. 

